Artículos científicos

\title{
Efecto de la poda de macrófitas sobre microorganismos adheridos al medio de soporte y la carga orgánica en humedales artificiales
}

\section{Effect of Macrophyte Pruning on Microorganisms Adhered to the Support Medium and the Organic Load in Artificial Wetlands}

Efeito da poda de macrófitas sobre os microrganismos aderidos ao meio de suporte e a carga orgânica em áreas úmidas artificiais

Mario José Romellón Cerino

Tecnológico Nacional de México, Instituto Tecnológico de Villahermosa, México mario.rc@villahermosa.tecnm.mx https://orcid.org/0000-0002-8579-1280

Gaspar López Ocaña

Universidad Juárez Autónoma de Tabasco, División Académica de Ciencias Biológicas,

México ocanag177@hotmail.com https://orcid.org/0000-0002-8402-8160

José Reyes Osorio Tecnológico Nacional de México, Instituto Tecnológico de Villahermosa, México jreyeso@itvillahermosa.edu.mx https://orcid.org/0000-0002-1313-3713

Julio Cesar Romellón Cerino

Tecnológico Nacional de México, Instituto Tecnológico de Villahermosa, México jcromellon@gmail.com https://orcid.org/0000-0003-2388-3128 
Revista Iberoamericana de las Ciencias Biológicas y Agropecuarias

Víctor Manuel Arias Peregrino

Tecnológico Nacional de México, Instituto Tecnológico de Villahermosa, México victor.ap@villahermosa.tecnm.mx https://orcid.org/0000-0001-5925-4097

\section{Resumen}

Esta investigación evaluó el efecto de la poda de macrófitas sobre microorganismos adheridos al medio de soporte y la remoción de $\mathrm{DBO}_{5}$ en tres humedales artificiales de flujo subsuperficial (HAFS) experimentales con especies de Phragmites australis (HAFSCarrizo), Pontederia sagittata (HAFS-Tule) y grava como testigo (HAFS-Grava). Se utilizaron nueve unidades (con réplicas). Los HAFS con $1.2 \mathrm{~m}$ de ancho, $2.5 \mathrm{~m}$ de largo y $0.5 \mathrm{~m}$ de profundidad se alimentaron con $200 \mathrm{~L} / \mathrm{d}$ agua residual doméstica. Las variables estudiadas al medio de soporte fueron microorganismos adheridos, porosidad, densidad, diámetro y humedad para estimar el tiempo de retención y conductividad hidráulica. En las especies se midieron las variables de productividad después de la poda y se estimó la remoción y el coeficiente cinético de degradación k para la $\mathrm{DBO}_{5}$. El experimento fue un diseño de un factor (tratamiento) con tres niveles (HAFS-Tule, HAFS-Carrizo y HAFSGrava); se evaluaron durante tres meses, después del año de operación. Los tratamientos se evaluaron mediante Anova (paramétricos) y la prueba de rangos de Kruskal-Wallis (no paramétricos) entre grupos antes y después de podar $(p<0.05)$. La grava presentó en promedio $(N=12) 22.92 \pm 1.47 \%$ de humedad, $2.32 \pm 0.15 \mathrm{~cm}$ de diámetro, densidad de partícula $2.71 \pm 0.10 \mathrm{gr} / \mathrm{cm}^{3}$ y porosidad de $36.52 \pm 3.36 \%$, el TRH fue de 3.1 días en todos los tratamientos. El mejor tratamiento fue HAFS-Tule: antes de la poda la biomasa de microorganismos presentó valores medianos $(N=12)$ de $42931.6 \mathrm{mg} / \mathrm{kg}\left(\mathrm{Q}_{1}=40259.7\right.$; $\left.\mathrm{Q}_{3}=54478.4\right)$ y posterior a la poda de $33444.6 \mathrm{mg} / \mathrm{kg}\left(\mathrm{Q}_{1}=31210.9 ; \mathrm{Q}_{3}=36581.8\right)$, la biomasa vegetal retirada en la poda fue de $40.85 \pm 2.58 \mathrm{~kg}$, se removió $95.44 \%$ de $\mathrm{DBO}_{5}$ con una $k=1.004$ días $^{-1}\left(27.6{ }^{\circ} \mathrm{C}\right)$, lo que permite cumplir la NOM-001-Semarnat-1996.

Palabras clave: densidad de partícula, humedal artificial de flujo subsuperficial, Phragmites australis, Pontederia sagittata, porosidad. 


\section{Abstract}

This research evaluated the effect of macrophyte pruning on microorganisms adhered to the support medium and the removal of $\mathrm{BOD}_{5}$ in three experimental subsurface flow artificial wetlands (FFSCW) with species of Phragmites australis (FFSCW-Carrizo), Pontederia sagittata (FFSCW-Tule) and gravel as a control (FFSCW-Grava), being nine units (with replicas). The FFSCW $1.2 \mathrm{~m}$ wide, $2.5 \mathrm{~m}$ long and $0.5 \mathrm{~m}$ deep were fed with $200 \mathrm{~L} / \mathrm{d}$ domestic wastewater. The variables studied to the support medium were adhered microorganisms, porosity, density, diameter and humidity to estimate the retention time and hydraulic conductivity. In the species, the productivity variables were measured after pruning and the removal and the degradation kinetic coefficient $\mathrm{k}$ for the $\mathrm{BOD}_{5}$ were estimated. The experiment was a one-factor (treatment) design with three levels (FFSCW-Tule, FFSCWCarrizo and FFSCW-Grava); being evaluated during three months, after the year of operation. Treatments were evaluated by ANOVA (parametric) and the Kruskal-Wallis rank test (non-parametric) between groups before and after pruning $(p<0.05)$. The gravel presented on average $(N=12) 22.92 \pm 1.47 \%$ humidity, $2.32 \pm 0.15 \mathrm{~cm}$ in diameter, particle density $2.71 \pm 0.10 \mathrm{gr} / \mathrm{cm}^{3}$ and porosity of $36.52 \pm 3.36 \%$, the TRH was 3.1 days in all the treatments. The best treatment was FFSCW-Tule, before pruning the biomass of microorganisms presented median values $(N=12)$ of $42931.6 \mathrm{mg} / \mathrm{kg} \quad\left(\mathrm{Q}_{1}=40259.7\right.$; $\left.\mathrm{Q}_{3}=54478.4\right)$ and after pruning of $33444.6 \mathrm{mg} / \mathrm{kg}\left(\mathrm{Q}_{1}=31210.9 ; \mathrm{Q}_{3}=36581.8\right)$, the vegetable biomass removed in pruning was $40.85 \pm 2.58 \mathrm{~kg}$, removing $95.44 \%$ of $\mathrm{BOD}_{5}$ with $k=1.004$ days $^{-1}\left(27.6^{\circ} \mathrm{C}\right)$, which allows complying with NOM-001-Semarnat-1996.

Keywords: particle density, subsurface-flow constructed wetland, Phragmites australis, Pontederia sagittata, porosity.

\section{Resumo}

Esta pesquisa avaliou o efeito da poda de macrófitas sobre microrganismos aderidos ao meio de suporte e a remoção de BOD5 em três pântanos experimentais de fluxo subsuperficial (HAFS) com espécies de Phragmites australis (HAFS-Carrizo), Pontederia sagittata (HAFSTule) e cascalho como controle (HAFS-Grava). Foram utilizadas nove unidades (com réplicas). Os HAFS de 1,2 $\mathrm{m}$ de largura, 2,5 $\mathrm{m}$ de comprimento e 0,5 $\mathrm{m}$ de profundidade foram alimentados com 200 L / d de águas residuais domésticas. As variáveis estudadas para 
o meio suporte foram microrganismos aderidos, porosidade, densidade, diâmetro e umidade para estimar o tempo de retenção e a condutividade hidráulica. Na espécie, as variáveis de produtividade foram medidas após a poda e estimados a remoção e o coeficiente cinético de degradação k para o DBO5. O experimento foi um projeto de um fator (tratamento) com três níveis (HAFS-Tule, HAFS-Carrizo e HAFS-Grava); eles foram avaliados por três meses, após o ano de operação. Os tratamentos foram avaliados por Anova (paramétrico) e teste de classificação Kruskal-Wallis (não paramétrico) entre os grupos antes e após a poda (p <0,05). A brita apresentou em média $(\mathrm{N}=12) 22,92 \pm 1,47 \%$ de umidade, 2,32 $\pm 0,15 \mathrm{~cm}$ de diâmetro, densidade de partícula $2,71 \pm 0,10 \mathrm{gr} / \mathrm{cm} 3$ e porosidade de $36,52 \pm 3,36 \%$, o TRH foi de 3,1 dias em todos os tratamentos. O melhor tratamento foi HAFS-Tule: antes da poda a biomassa dos microrganismos apresentou valores medianos $(\mathrm{N}=12)$ de $42931,6 \mathrm{mg} / \mathrm{kg}$ $(\mathrm{Q} 1=40259,7 ; \mathrm{Q} 3=54478,4)$ e após a poda de 33444,6 mg / kg $(\mathrm{Q} 1=31210,9$; Q3 = 36581,8 ), a biomassa vegetal removida na poda foi de 40,85 $\pm 2,58 \mathrm{~kg}, 95,44 \%$ do BOD5 foi removido com $\mathrm{k}=1.004$ dias- $1\left(27,6^{\circ} \mathrm{C}\right)$, o que permite cumprir a NOM-001 -Semarnat1996.

Palavras-chave: densidade de partículas, pântano de fluxo subsuperficial artificial, Phragmites australis, Pontederia sagittata, porosidade.

Fecha recepción: Mayo 2021

Fecha aceptación: Diciembre 2021

\section{Introducción}

Las aguas residuales son típicamente mezclas de compuestos orgánicos e inorgánicos clasificados como contaminantes básicos y xenobióticos tóxicos, entre otros (Miranda, Sandoval, Calvo, Moeller y Sarracino, 2017). En los últimos años, se ha buscado minimizar y valorizar los lodos (por las características arriba mencionadas, residuos peligrosos tanto para el medio ambiente como para los seres vivos) que se generan a raíz del tratamiento de las aguas residuales, cuyas cantidades se han incrementado por el crecimiento poblacional y el desarrollo de las grandes urbes, por ejemplo, como materia prima para biocombustibles y mezclas para la formación de ladrillos (Araujo, Molina y Noguera, 2018; Mancipe y Triviño, 2018). De ahí la necesidad de aplicar alternativas tecnológicas de tratamiento de aguas residuales que sean de bajo costo (inversión-operación), de fácil operación y mantenimiento y con bajo consumo energético (Torres, López, Romellón, Vázquez y Comparán, 2020). Una 
Revista Iberoamericana de las Ciencias Biológicas y Agropecuarias

de estas alternativas son los humedales artificiales (HA). Los HA son estanques en extensiones de terreno con medio de soporte, agua residual, microorganismos y vegetación macrófita (Conagua, 2016).

Un humedal artificial de flujo subsuperficial (HAFS) es diseñado específicamente para el tratamiento de algún tipo de agua residual y está construido en forma de un lecho donde la grava presenta una altura hasta de 0.6 m (Asprilla, Ramírez y Rodriguez, 2020). El tratamiento se fundamenta en tres principios básicos: la actividad bioquímica de microorganismos, el aporte de oxígeno a través de las macrófitas durante el día y el apoyo físico de un lecho que sirve como soporte de microorganismos, enraizamiento de las especies y como material filtrante, elementos que, en conjunto, eliminan materiales disueltos y suspendidos en el agua residual (García, López y Torres, 2019).

Se ha reportado que el tipo de humedal, los tiempos de retención hidráulica (TRH) y las especies presentan afinidad para remover algunos contaminantes. Castañeda y Flores (2013) evaluaron HA experimentales con especies de humedales naturales de la región de Los Altos de Jalisco como son: el carrizo común (Phragmites australis), el gladiolo (Gladiolus spp) y la totora (Typha latifolia). Los HA operaron con TRH de tres, cinco y siete días; se realizaron cuatro monitoreos al año, y las mejores eficiencias fueron con siete días. La remoción de $\mathrm{DBO}_{5}$ con carrizo fue de $80.45 \%$, con gladiolo de $63.31 \%$ y con totora de $77.19 \%$. Para nitrógeno total (NT) con carrizo fue de $64 \%$, con gladiolo de $34 \%$ y con totora de $53 \%$. Para fósforo total (PT) el carrizo removió $65 \%$, con el gladiolo $68 \%$ y con totora $75.75 \%$. En estos resultados se observa que Phragmites australis fue mejor en la remoción de $\mathrm{DBO}_{5}$ y NT, mientras que el PT logró mejor remoción con Typha latifolia.

Recientemente, Rangel et al. (2019) utilizaron macrófitas Eichhornia crassipes y Typha domingensis para optimizar la cinética de degradación de la materia orgánica y eliminación de nutrientes en HA a escala laboratorio con TRH de dos y cuatro días. Se logró remover $92.39 \%$ de DQO, $99.28 \%$ de N y $87.78 \%$ de P con E. crassipes con cuatro días de TRH. El estudio cinético fue realizado comparando tres modelos (modelo de primer orden, modelo de Stover-Kincannon y modelo de Grau-segundo orden) y el mejor ajuste se obtuvo con el modelo cinético de Stover-Kincannon, con una $k=0.9997$ y una tasa máxima de remoción de $2500 \mathrm{mg} / \mathrm{Ld}$. 
Por su parte, Cui et al. (2011) estudiaron en China la eliminación de contaminantes en HAFS según el modelo cinético de primer orden. Estos autores reportan que la carga de masa del efluente se correlaciona con la temperatura en cierta medida. Estimaron que el coeficiente de los SST es de 0.6293 y para la DQO de 0.6210. La constante de tasa de área de SST aumenta exponencialmente con el aumento de la carga de masa del influente cuando la carga de masa es menor a $25 \mathrm{mg} / \mathrm{L}$, pero cambia mucho con una carga de masa más alta. Consideran que las condiciones ambientales de Beijing influyen en las constantes cinéticas y son más altas en otoño en comparación con las de verano e invierno. En otros estudios se ha planteado que la capacidad de tratamiento en HA es alta en las áreas tropicales debido a las temperaturas cálidas y a tasas más altas de actividad microbiana, por ejemplo, en Vietnam, se estudiaron HAFS con Phragmites vallatoria (L.) y se estimaron que las constantes de tasa de remoción de primer orden basadas en el área $\left(k, \mathrm{~m}_{\text {año }}{ }^{-1}\right)$ (a lo largo del humedal desde la entrada hasta la salida) en cuatro HA presentaron efluentes con rangos de 25-95 (DBO5), 2230 (DQO), removiendo 65-83 \% y 57-84 \%, respectivamente (Ngo et al., 2011).

Las especies Carex appressa, Ficinia nodosa y un control fueron utilizadas en biofiltros de columnas experimentales para conocer los efectos de la poda en la remoción de NT, PT y metales. El efluente se monitoreó en columnas de control podadas, no podadas y no plantadas durante 70 días, con un muestreo mensual de agua compuesta que abarca el agua de la zona saturada enjuagada y el efluente de cada columna. No se encontraron diferencias entre los tratamientos para el PT, pero los tratamientos de poda afectaron la eliminación de $\mathrm{NO}$ en fechas de muestreo posteriores para $F$. nodosa, pero no para $C$. appressa. La eliminación de N y P varió de 77-88 \% y 66-93 \%, respectivamente, tanto por plantas podadas como no podadas. La cantidad de $\mathrm{N}$ y $\mathrm{P}$ eliminados en la biomasa podada fue de 2.1 a 3.5 veces mayor que la cantidad estimada eliminada del afluente por el rebrote de las columnas podadas solo durante el periodo de rebrote. En consecuencia, la cantidad de nutrientes eliminados a través de la poda puede afectar significativamente la eliminación a largo plazo. Las concentraciones de efluentes de cadmio, cobre, plomo y zinc fueron similares entre los tratamientos con remoción superior a $95 \%$. En general, la poda parece afectar la mejora de la calidad del agua, pero las prácticas óptimas de poda que pueden mejorar la eliminación a largo plazo (Herzog et al., 2021). 
Ahora bien, el uso de humedales ha incrementado paulatinamente en el sureste de México. Para el 2019, en Tabasco se reportó una capacidad instalada de tratamiento de 2775.9 L/s atendidas con 69 plantas de tratamiento de aguas residuales, de las cuales 11 cuentan con HA (primario-secundario-terciario), por medio de las cuales se trata $26.57 \%$ (1015.3 L/s) del agua residual generada en el estado (Conagua-Semarnat, 2019). Dentro de los principales problemas operativos que se reportan son la colmatación en los primeros años de operación, lo que disminuye la remoción de los contaminantes básicos, y la especie más utilizada es Tyhpa domingensis (espadaño). Las especies desde que se siembran tienen poco mantenimiento (poda o resiembra), por lo que desarrollan su ciclo de vida y es común que presenten variación en la eficiencia de remoción y altas concentraciones en efluente, lo que provoca a su vez el fenómeno de corto circuito (Vázquez y López, 2011).

En el presente estudio se evaluó el efecto de la poda en dos especies macrófitas (Pontederia sagittata y Phragmites australis) sobre los microorganismos del soporte y en la remoción de $\mathrm{DBO}_{5}$. Dicho estudio se realizó después de que los humedales cumplieron un año de operación (arranque y estabilización); operaron de forma normal y la poda fue parte del mantenimiento preventivo. Los HA se monitorearon durante tres meses para identificar los posibles cambios que sufre el medio de soporte en cuanto a los microorganismos y demás variables físicas. A la par, se evaluó la remoción y cinética de $\mathrm{DBO}_{5}$ de cada especie en los HAFS. Esta investigación considera como hipótesis que existe un efecto de la poda sobre los microorganismos y como consecuencia se altera la remoción de materia orgánica medida como $\mathrm{DBO}_{5}$ en los HAFS.

\section{Materiales y métodos}

\section{Unidades experimentales}

El agua residual utilizada en los HAFS fue del cárcamo concentrador de la Universidad Juárez Autónoma de Tabasco (UJAT), en específico de la División Académica de Ciencias Biológicas (DACBiol), usada en servicios sanitarios y cafeterías. Se utilizaron nueve unidades experimentales de HAFS de manera independiente diseñados por López et al. (2014). Se usaron dos bombas de $1 \mathrm{HP}$, conectadas a tubería hidráulica de 1". La tubería de PVC se conectó a tres tanques que regulan el flujo (200 L). Cada HAFS mantuvo el mismo caudal (200 L/día), velocidad de entrada y carga orgánica. Además, cada una de ellas estuvo 
compuesta de acero al carbón calibre 10, de 2.5 m largo x 1.2 m ancho x 1 m alto. Se aplicó esmalte alquidálico anticorrosivo y un epóxico catalizado con poliamidas para proteger la unidad. Internamente se aplicó una capa de impermeabilizante elastomérico con capas de fibra textil, rellenándose con grava mixta $\left(\varnothing \mathrm{de} 2.25 \mathrm{~cm}\right.$; densidad de $2.65 \mathrm{gr} / \mathrm{cm}^{3}$; porosidad de $35.81 \%$ ), con una altura de $0.5 \mathrm{~m}$ de tirante operativo. La operación de las unidades experimentales inicia con la captación de agua cruda proveniente de sanitarios y cafeterías en el cárcamo cisterna, que funciona como tratamiento primario al cumplir con funciones de remoción de residuos sólidos urbanos, sólidos sedimentables y suspendidos con TRH de 45 a 250 minutos; enseguida, se bombea el agua a tanques de control, donde se regula el gasto y velocidad, y posteriormente pasa a las unidades experimentales que están operando como tratamiento secundario (figura 1).

Figura 1. Diagrama de especificaciones de las unidades experimentales instaladas

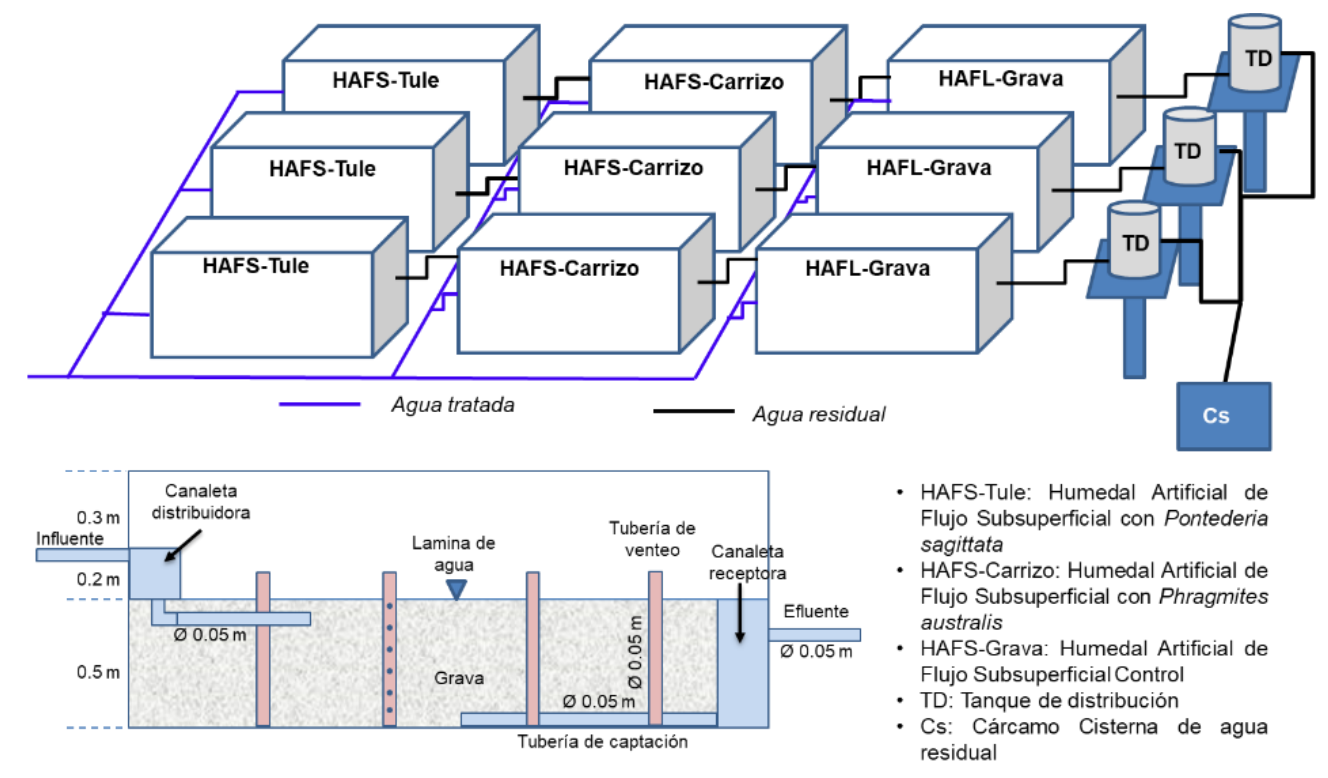

Fuente: Elaboración propia

\section{Colecta de especies macrófitas}

El muestreo de vegetación macrófita Pontederia sagittata y Phragmites australis fue obtenido de la Reserva de la Biosfera Pantanos de Centla, donde fueron colectadas y transportadas siguiendo las recomendaciones de Gallegos, López, Bautista y Torres (2018). Dicha colecta fue en agosto de 2018. Una vez que las plantas se encontraron en el área del experimento, se colocaron en un canal de descarga de agua residual tratada. La plantación se 
Revista Iberoamericana de las Ciencias Biológicas y Agropecuarias

efectuó al día siguiente para su estabilización. La distribución de especies en el sembrado de Pontederia sagittata dentro de los tres humedales fue de 15 plantas para cada uno. Mientras que de Phragmites australis se sembraron 20 surcos en cada uno de los tres humedales, cada surco constó de cuatro plantas. Los humedales control (HAFS-Grava) solo contenían $0.5 \mathrm{~m}$ de grava. La especie Pontederia sagittata tuvo rápida adaptación y aceptación del agua residual, ya que entre los primeros 8 y 10 días se observó el crecimiento foliar en la parte central, mientras que Phragmites australis mostró adaptación entre los 20 y 25 días, cuando se observó el crecimiento de pequeñas raíces y yemas en los nudos del tallo. Se trata de una pequeña diferencia respecto a otras especies que son adaptadas con agua reconstituida antes de plantar. Se observó que se necesitaron aproximadamente 40 días para que existiera estabilización.

\section{Monitoreo del medio de soporte, las macrófitas y la $\mathrm{DBO}_{5}$}

Las variables de respuesta medidas al medio de soporte y a las macrófitas fueron: la humedad (Hum), el diámetro de grava (Øp) y la densidad de la partícula (Dp), conforme lo establece la NOM-021-RECNAT-2000, la porosidad (y), por el método establecido por Muñoz, Soler, López y Hernádez (2015), y la biomasa de microorganismos, por el método modificado de materia volátil de López et al. (2019). Y a las especies se le determinó biomasa vegetal $(\mathrm{kg})$, altura $(\mathrm{m})$, largo hoja $(\mathrm{cm})$, ancho hoja $(\mathrm{cm})$, diámetro tallo $(\mathrm{cm})$ y largo raíz (cm) (Gallegos et al., 2018). Estas determinaciones se realizaron en operación normal después de un año de funcionamiento. Se midieron en el mes de octubre 15 días antes de la poda ( 1 al 15 de octubre) y 15 días después de la poda (15 al 30 de octubre). Y se tomaron tres muestras a lo largo y ancho de cada HA, con una distancia promedio de $0.35 \mathrm{~m}, 1.25 \mathrm{~m}$ y $2.15 \mathrm{~m}$ a partir de la entrada hasta la salida del efluente; en total, 9 muestras del medio de soporte por humedal. Esto para medir el efecto inmediato de la poda sobre los microorganismos y las especies. A las especies podadas el 15 de octubre se les dejó un seudotallo de $15 \mathrm{~cm}$ sobre el medio de soporte y pesaron, tomando tres plantas a lo largo y ancho del HA, de donde se midieron las variables mencionadas anteriormente. La medición de $\mathrm{DBO}_{5}$ se realizó un día a la semana (12 muestras compuestas) durante un periodo de tres meses (septiembre-noviembre 2019); así, se obtuvieron muestras simples cada tres horas desde las 8:00 h y las 17:00 h, y se determinó mediante el método NMX-AA-028-SCFI-2001. Cabe aclarar que seis muestras están dentro del periodo antes de la poda y seis muestreos son 
posterior a la poda. La poda se recomienda realizar por lo menos dos veces al año (Conagua, 2016).

\section{Diseño experimental y análisis estadístico}

Se empleó un diseño de un factor (tratamiento) con tres niveles (HAFS-Tule, HAFSCarrizo y HAFS-Grava). El experimento se corrió por triplicado. El periodo de monitoreo fue de tres meses (septiembre, octubre y noviembre 2019) después de un año de operación. Para determinar diferencias estadísticamente significativas en la evaluación del sistema de HA entre los grupos de tratamientos, se contrastó con un análisis de varianza (Anova) de una clasificación por rangos para los datos que no cumplieron con postulados de normalidad y homocedasticidad de Kruskal-Wallis, así como también con un contraste de medianas para muestras independientes U Mann-Whitney (antes de la poda: biomasa de microorganismos, diámetro de partículas y densidad de la grava; después de la poda: biomasa de microorganismos y humedad). Para los datos que cumplieron con los postulados de normalidad y homocedasticidad, se les realizó una prueba Anova (antes de la poda: humedad y porosidad; después de la poda: diámetro de partícula, densidad de la grava y porosidad).

\section{Tiempo de retención hidráulica}

El tiempo de retención hidráulico se determinó para los gastos de operación mediante la ecuación 1 (Crites y Tchobanoglous, 2000):

$$
T R H=\frac{n h A s}{Q}
$$

Donde: TRH $=$ Tiempo de retención hidráulica, $n=$ Porosidad $h=$ Profundidad de la lámina del agua, $A s=$ Área del humedal y $Q=$ Gasto promedio.

En la prueba hidráulica, una vez conocido el volumen del reactor con el tirante operativo, el volumen de grava y la porosidad, se realizaron pruebas suministrando diferentes gastos de operación (50 a 350 L/día) con la finalidad de conocer el TRH a diferentes gastos

y así estimar el gasto de operación de las unidades experimentales. Se estableció que todo el experimento se desarrollaría con un caudal de 200 L/día. 


\section{Eficiencia de remoción}

La eficiencia de remoción para $\mathrm{DBO}_{5}$ se calculó mediante la siguiente ecuación 2 (García et al., 2019):

$$
E R(\%)=\frac{c_{e}-c_{s}}{c_{e}} \times 100
$$

Dónde: $\mathrm{ER}(\%)=$ Eficiencia de remoción $(\%), \mathrm{Ce}=$ Concentración de entrada (influente) y $\mathrm{Cs}=$ Concentración de salida (efluente).

\section{Cinética de degradación}

Debido a que los sistemas de HA son reactores biológicos, su rendimiento puede ser estimado mediante una cinética de primer orden (Cui et al., 2011; Environmental Protection Agency [EPA], 2000). La constante de reacción k se estimó mediante la ecuación 3. En el caso de nuestro estudio, los experimentos fueron desarrollados a temperatura de $27.63{ }^{\circ} \mathrm{C}$ en promedio, por lo que ya no fue necesario realizar el ajuste de Arrhenius, como lo establece Crites y Tchobanoglous, (2000).

$$
c_{e}=c_{o} e^{-k t}
$$

Dónde: $\mathrm{Ce}=$ Concentración del contaminante en el efluente $(\mathrm{mg} / \mathrm{L})$, $\mathrm{Co}=$ Concentración del contaminante en el influente $(\mathrm{mg} / \mathrm{L}), k=$ Constante de reacción $\left(\mathrm{d}^{-1}\right)$ y $t=$ Tiempo de retención hidráulica (día).

\section{Resultados}

A continuación, se muestran los resultados obtenidos de los tratamientos. Cabe recordar que hubo mediciones antes y después de podar las especies durante el periodo de experimentación, parámetros físicos y biomasa bacteriana en el medio de soporte. Del mismo modo, se estudió la remoción y cinética de degradación de la $\mathrm{DBO}_{5}$, así como las características de las especies (largo y ancho de hoja, altura, diámetro del tallo y longitud de raíz) después de la poda. La concentración inicial del agua de entrada a los experimentos 
presentó los promedios de los siguientes parámetros: $\mathrm{DBO}_{5} 408.1 \mathrm{mg} / \mathrm{L}, \mathrm{SST} 310.0 \mathrm{mg} / \mathrm{L}$, Turbiedad 131.4 NTU y Color 946.1 UC, pH 7.8 y temperatura del agua $27.6^{\circ} \mathrm{C}$.

\section{Condiciones antes de la poda}

\section{Biomasa de microorganismos}

Los resultados de Kruskal-Wallis, para el contraste de medianas en la biomasa de microorganismos $(\mathrm{mg} / \mathrm{kg})$ en el medio de soporte de los diferentes HA (HAFS-Carrizo, HAFS-Tule y HAFS-Grava) antes de la poda, indican que existen diferencias estadísticamente significativas entre los tratamientos $(p<0.01)$. La prueba de muestras independientes U Mann-Whitney indicó la presencia de diferencias estadísticas significativas en ambas medianas de los tratamientos $(p<0.05)$. El valor mediano más bajo observado fue en el HAFS-Carrizo, $39340.5 \mathrm{mg} / \mathrm{kg}$ con cuartiles inferior y superior $\left(\mathrm{Q}_{1}=37355.2 ; \mathrm{Q}_{3}=\right.$ 54329.1), seguido del HAFS-Tule con $42931.6 \mathrm{mg} / \mathrm{kg}\left(\mathrm{Q}_{1}=40259.7 ; \mathrm{Q}_{3}=54478.4\right)$, por lo tanto, el HAFS-Grava obtuvo el valor mediano más alto con $52179.6 \mathrm{mg} / \mathrm{kg}\left(\mathrm{Q}_{1}=44789.5\right.$; $\mathrm{Q}_{3}=74419.8$ ) (figura 2).

Figura 2. Valores de medianas $\left( \pm \mathrm{Q}_{1,3}\right)$ para biomasa de microorganismos $\mathrm{mg} / \mathrm{kg}$

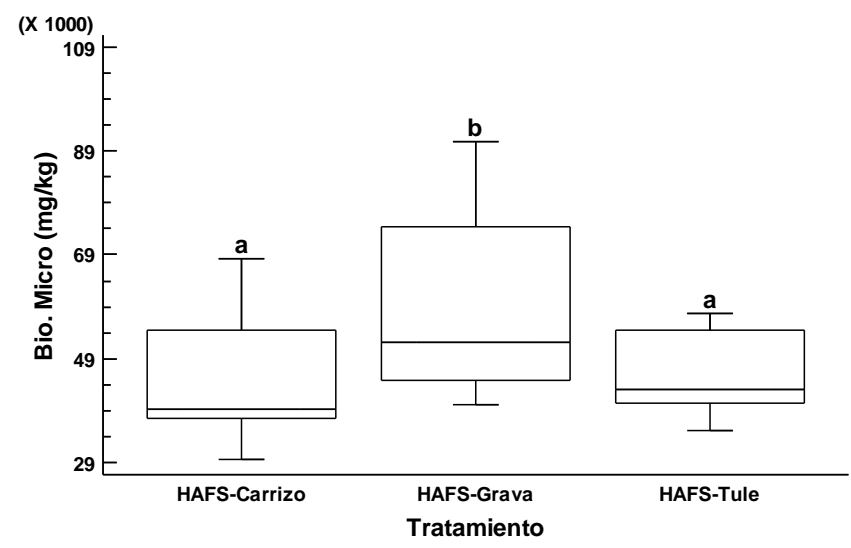

Nota: letras diferentes indican diferencias estadísticamente significativas $(N=12)$

Fuente: Elaboración propia

En el análisis de la distribución de biomasa de microorganismos dentro de los humedales se encontró la mayor adhesión en la entrada del agua residual, disminuyendo gradualmente hacia la salida del humedal (figura 3). Las concentraciones medianas de biomasa de microorganismos en la entrada para el HAFS-Tule a la distancia de $0.35 \mathrm{~m}$ fueron de $56498.08 \mathrm{mg} / \mathrm{kg}\left(\mathrm{Q}_{1}=54927.35 ; \mathrm{Q}_{3}=57650.22\right)$, disminuyendo a la salida en 41695.60 
$\mathrm{mg} / \mathrm{kg}\left(\mathrm{Q}_{1}=39214.34 ; \mathrm{Q}_{3}=42862.75\right)$ en la distancia de $2.15 \mathrm{~m}$. Para el HAFS-Carrizo se encontró un dato de $56270.08 \mathrm{mg} / \mathrm{kg}\left(\mathrm{Q}_{1}=54350.77 ; \mathrm{Q}_{3}=60701.09\right)$, disminuyendo a la salida en $33295.33 \mathrm{mg} / \mathrm{kg}\left(\mathrm{Q}_{1}=30363.34 ; \mathrm{Q}_{3}=36656.01\right)$. Mientras que para el HAFS-Grava la concentración de entrada fue $76917.52 \mathrm{mg} / \mathrm{kg}\left(\mathrm{Q}_{1}=74653.74 ; \mathrm{Q}_{3}=81902.83\right)$ disminuyendo a la salida en $42654.63 \mathrm{mg} / \mathrm{kg}\left(\mathrm{Q}_{1}=41366.98 ; \mathrm{Q}_{3}=46394.18\right)$ respectivamente.

Figura 3. Distribución de microorganismos $(\mathrm{mg} / \mathrm{kg})$ en la sección longitudinal del HAFS

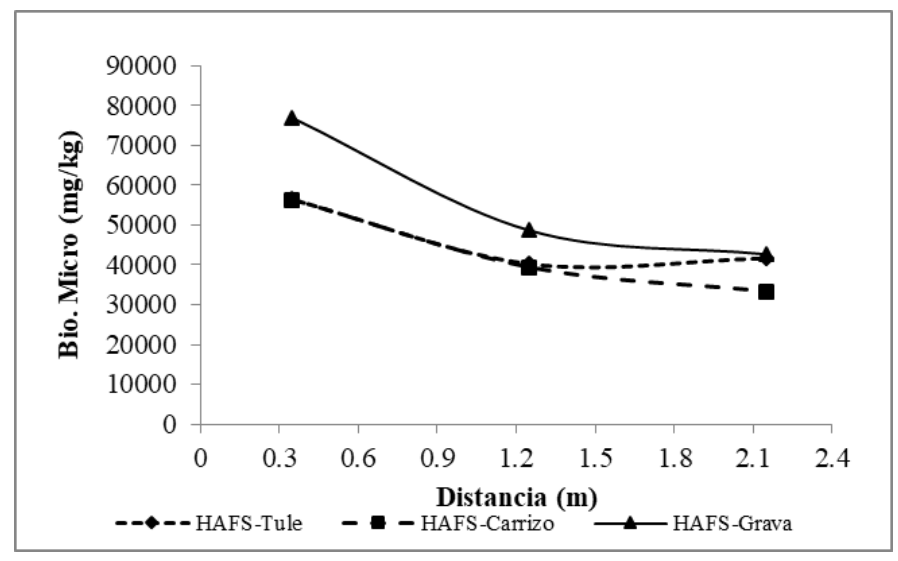

Fuente: Elaboración propia

\section{Humedad}

Los resultados del Anova en la comparación de los valores promedios de la humedad (\%) en el medio de soporte de los distintos HA (HAFS-Tule, HAFS-Carrizo y HAFS-Grava), indican que no existen diferencias estadísticas significativas entre los tratamientos (Anova, $\mathrm{F} 2.33=1.55, p>0.05)$. El valor promedio más alto de humedad se obtuvo en el HAFS-Grava con $24.56 \pm 0.81 \%$, seguido del HAFS-Carrizo con $24.23 \pm 0.69 \%$, mientras que la media más baja fue para el tratamiento del HAFS-Tule con $23.89 \pm 1.20 \%$ (figura 4 ). 
Figura 4. Valores promedios $( \pm \mathrm{EE})$ en la humedad $(\%)$

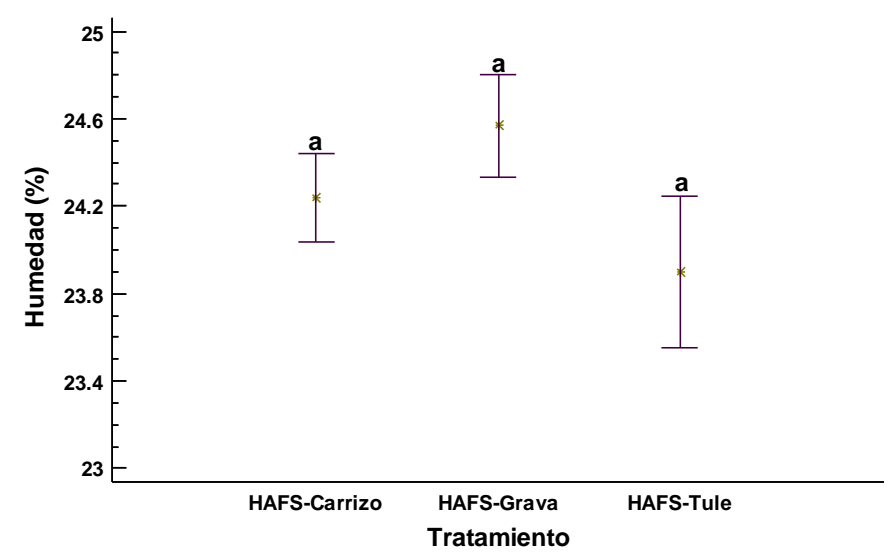

Nota: letras diferentes indican diferencias estadísticamente significativas $(N=12)$.

Fuente: Elaboración propia

\section{Diámetro de partícula (grava)}

El análisis de Kruskal-Wallis, para el contraste de medianas del diámetro $(\mathrm{cm})$ medidos en el medio de soporte de los distintos HA (HAFS-Tule, HAFS-Carrizo y HAFSGrava), indica que no existen diferencias estadísticas significativas entre las medianas de cada uno de los tres niveles de tratamiento $(p>0.05)$. Por lo que el valor mediano del diámetro obtenido para el HAFS-Carrizo es $2.38 \mathrm{~cm}\left(\mathrm{Q}_{1}=2.23, \mathrm{Q}_{3}=2.60\right)$, el HAFS-Grava tuvo una mediana de $2.10 \mathrm{~cm}\left(\mathrm{Q}_{1}=2.03 ; \mathrm{Q}_{3}=2.33\right)$ y el HAFS-Tule obtuvo una mediana de $2.28 \mathrm{~cm}\left(\mathrm{Q}_{1}=2.10, \mathrm{Q}_{3}=2.30\right)($ figura 5$)$.

Figura 5. Valores de medianas $\left( \pm \mathrm{Q}_{1}, 3\right)$ del diámetro $(\mathrm{cm})$ del medio de soporte

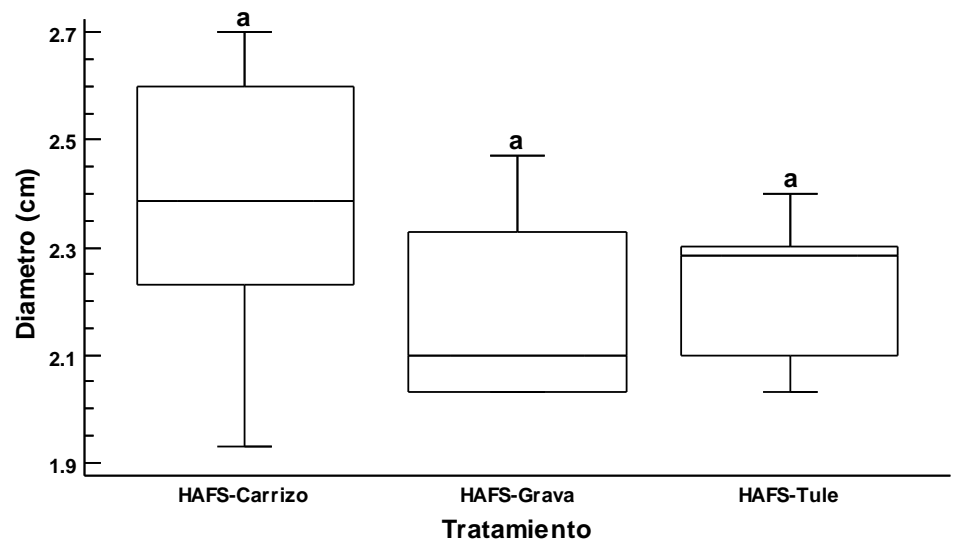

Nota: letras diferentes indican diferencias estadísticamente significativas $(N=12)$

Fuente: Elaboración propia 


\section{Densidad de partícula (grava)}

Para la densidad de la partícula (grava), los resultados de Kruskal-Wallis para el contraste de medianas determinados en el medio de soporte de los distintos HA (HAFS-Tule, HAFS-Carrizo y HAFS-Grava) indican que no existen diferencias estadísticas significativas entre las medianas de cada uno de los tres niveles de tratamiento $(p>0.05)$. La densidad de la partícula en el HAFS-Carrizo presenta una mediana de $2.60 \mathrm{gr} / \mathrm{cm}^{3}\left(\mathrm{Q}_{1}=2.52 ; \mathrm{Q}_{3}=2.66\right)$, mientras que el HAFS-Grava tiene una mediana de $2.77 \mathrm{gr} / \mathrm{cm}^{3}\left(\mathrm{Q}_{1}=2.68 ; \mathrm{Q}_{3}=2.85\right)$ y el HAFS-Tule presentó una mediana de $2.60 \mathrm{gr} / \mathrm{cm}^{3}\left(\mathrm{Q}_{1}=2.56\right.$; $\left.\mathrm{Q}_{3}=2.93\right)$ (figura 6).

Figura 6. Valores de medianas $\left( \pm \mathrm{Q}_{1,3}\right)$ en la densidad de la partícula $\left(\mathrm{gr} / \mathrm{cm}^{3}\right)$

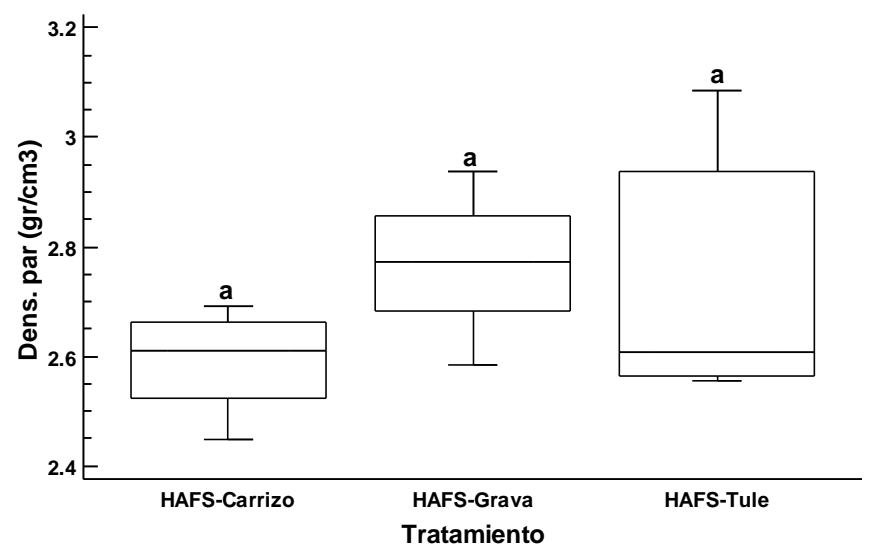

Nota: Letras diferentes indican diferencias estadísticamente significativas $(N=12)$

Fuente: Elaboración propia

\section{Porosidad}

Los resultados del Anova de comparación de la porosidad para los distintos HA (HAFS-Tule, HAFS-Carrizo y HAFS-Grava) indican que existe una diferencia estadística altamente significativa entre los tratamientos (Anova, F2.33 $=10.79, p<0.001$ ). La prueba a posteriori de contrastes múltiples de Tukey indicó la presencia de diferencias estadísticas significativas entre los tratamientos $(p<0.05)$. El valor promedio más alto de porosidad se obtuvo en el HAFS-Tule con $38.85 \pm 3.30 \%$, seguido del HAFS-Carrizo con $35.10 \pm 2.91 \%$, mientras que la media más baja fue para el tratamiento del HAFS-Grava con $33.48 \pm 2.41 \%$ (figura 7). 
Figura 7. Valores promedios $( \pm \mathrm{EE})$ de la porosidad $(\%)$

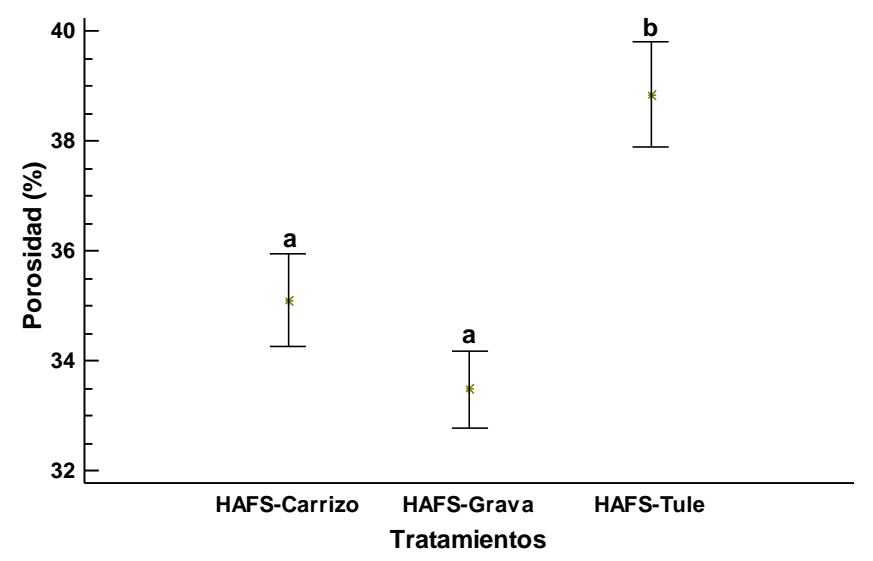

Nota: letras diferentes indican diferencias estadísticamente significativas $(N=12)$.

Fuente: Elaboración propia

\section{Condiciones después de la poda}

A continuación, se presentan los resultados de las características hidráulicas de los diferentes tratamientos (HAFS-Tule, HAFS-Carrizo y HAFS-Grava) obtenidos después de haber efectuado el corte de la vegetación.

\section{Biomasa de microorganismos}

Los resultados de Kruskal-Wallis para el contraste de medianas en medición de biomasa de microorganismos $(\mathrm{mg} / \mathrm{kg})$ en el medio de soporte de los diferentes HA (HAFSCarrizo, HAFS-Tule y HAFS-Grava) después de la poda indican que existen diferencias estadísticamente significativas entre los tratamientos $(p<0.01)$. La prueba de muestras independientes U Mann-Whitney indicó la presencia de diferencias estadísticas significativas en ambas medianas de los tratamientos $(p<0.05)$. El valor mediano más bajo observado fue el HAFS-Tule 33444.6 mg/kg $\left(\mathrm{Q}_{1}=31210.9 ; \mathrm{Q}_{3}=36581.8\right)$, seguido del HAFS-Carrizo $40489.7 \mathrm{mg} / \mathrm{kg}\left(\mathrm{Q}_{1}=35586.4 ; \mathrm{Q}_{3}=45830.4\right)$, por lo tanto, el HAFS-Grava obtuvo el valor mediano más alto de $61746.1 \mathrm{mg} / \mathrm{kg}\left(\mathrm{Q}_{1}=43753.1 ; \mathrm{Q}_{3}=71624.1\right)$ (figura 8). 
Figura 8. Valores de medianas $\left( \pm \mathrm{Q}_{1}, 3\right)$ para biomasa de microorganismos $(\mathrm{mg} / \mathrm{kg})$

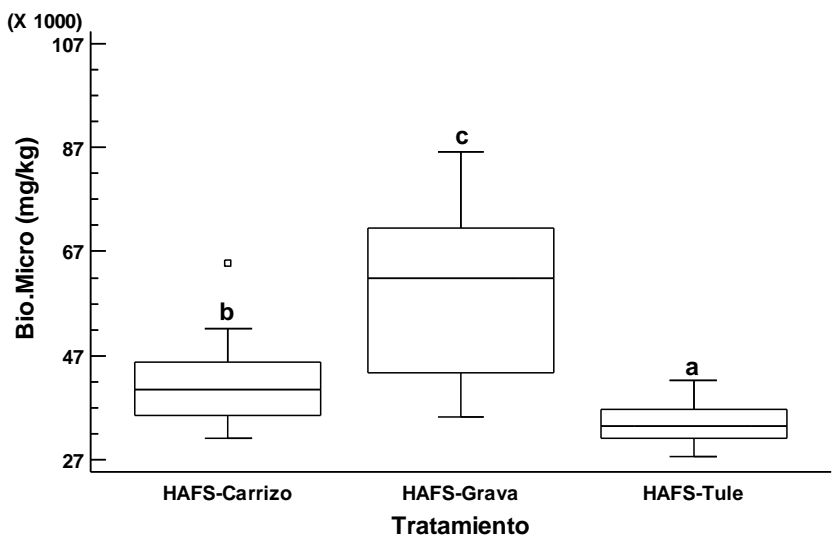

Nota: letras diferentes indican diferencias estadísticamente significativas $(N=12)$.

Fuente: Elaboración propia

El comportamiento mostrado después de la poda es similar al mostrado en la fase antes de la poda: disminuye gradualmente del influente al efluente (figura 9). Los valores de medianas de la biomasa de microorganismos determinadas en la entrada para el HAFS-Tule $(0.35 \mathrm{~m})$ fueron de $34971.82 \mathrm{mg} / \mathrm{kg}\left(\mathrm{Q}_{1}=31770.51 ; \mathrm{Q}_{3}=38627.77\right)$ disminuyendo a la salida en $32032.52 \mathrm{mg} / \mathrm{kg}\left(\mathrm{Q}_{1}=29910.73 ; \mathrm{Q}_{3}=33931.94\right)$ en la distancia de $2.15 \mathrm{~m}$. Para el HAFSCarrizo, la concentración inicial fue de $42152.11 \mathrm{mg} / \mathrm{kg}\left(\mathrm{Q}_{1}=38487.35 ; \mathrm{Q}_{3}=47348.75\right)$ disminuyendo a la salida con $34928.23 \mathrm{mg} / \mathrm{kg}\left(\mathrm{Q}_{1}=33643.46 ; \mathrm{Q}_{3}=37087.16\right)$. Mientras que para el HAFS-Grava la concentración inicial fue $71624.12 \mathrm{mg} / \mathrm{kg} \quad\left(\mathrm{Q}_{1}=66507.38\right.$; $\left.\mathrm{Q}_{3}=79117.46\right) \quad$ disminuyendo a la salida en $41717.19 \mathrm{mg} / \mathrm{kg} \quad\left(\mathrm{Q}_{1}=38667.33\right.$; $\left.\mathrm{Q}_{3}=43673.42\right)$. 
Figura 9. Distribución de la biomasa de microorganismos $(\mathrm{mg} / \mathrm{kg})$ en la sección longitudinal del humedal

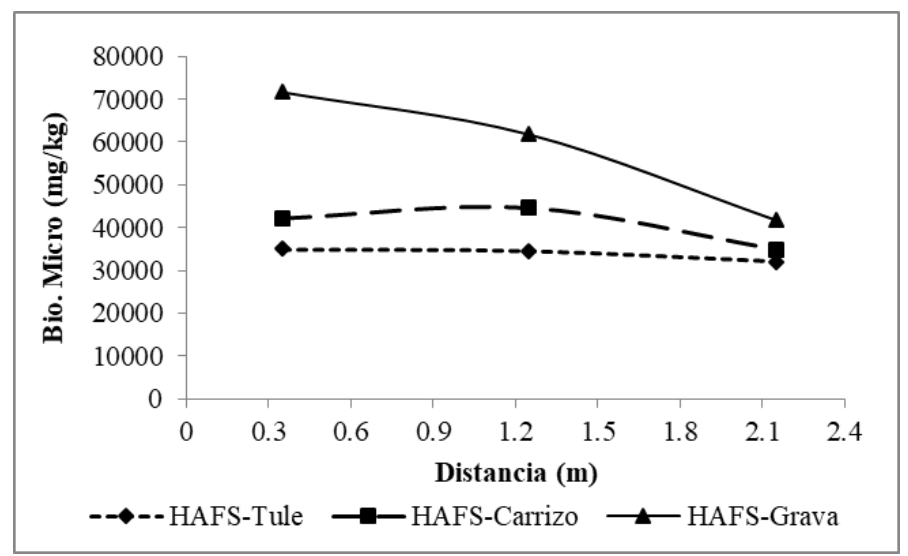

Fuente: Elaboración propia

\section{Humedad}

El análisis de Kruskal-Wallis, en la humedad (\%) del medio de soporte a los distintos HA (HAFS-Tule, HAFS-Carrizo y HAFS-Grava), indica que no existen diferencias estadísticamente significativas entre las medianas de cada uno de los tres niveles de tratamiento $(p>0.05)$. Por lo que el valor mediano de la humedad (\%) obtenido para el HAFS-Carrizo es $21.32 \%\left(\mathrm{Q}_{1}=20.21 ; \mathrm{Q}_{3}=22.63\right)$, el HAFS-Grava tuvo una mediana de $21.15 \%\left(\mathrm{Q}_{1}=20.22 ; \mathrm{Q}_{3}=21.74\right)$ y el HAFS-Tule obtuvo una mediana de $22.19 \%$ $\left(\mathrm{Q}_{1}=21.43 ; \mathrm{Q}_{3}=23.63\right)$ (figura 10).

Figura 10. Valores de medianas $\pm \mathrm{Q}_{1}, 3$ en la humedad (\%)

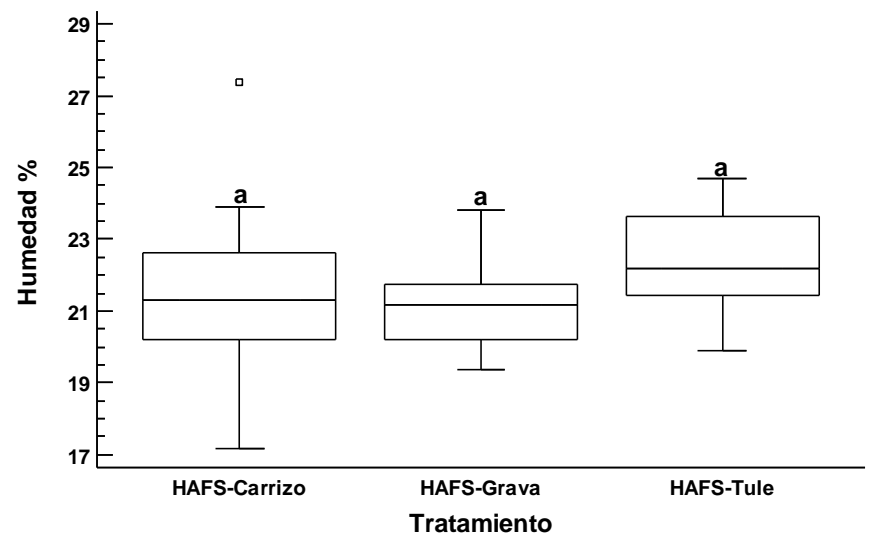

Nota: letras diferentes indican diferencias estadísticamente significativas $(N=12)$

Fuente: Elaboración propia 


\section{Porosidad}

Los resultados del Anova indican que existe una diferencia estadística altamente significativa entre los tratamientos (Anova, $\mathrm{F} 2.33=33.39, p<0.001$ ). La prueba $a$ posterior $i$ de contrastes múltiples de Tukey indicó la presencia de diferencias estadísticas significativas entre los tratamientos $(\mathrm{p}<0.05$ ). El valor promedio más alto de porosidad se obtuvo en el HAFS-Tule con $40.85 \pm 2.90 \%$, seguido del HAFS-Carrizo con $38.46 \pm 2.53 \%$, mientras que la media más baja fue para el tratamiento del HAFS-Grava con $32.38 \pm 2.39 \%$ (figura $11)$.

Figura 11. Valores promedios $( \pm \mathrm{EE})$ de la porosidad $(\%)$

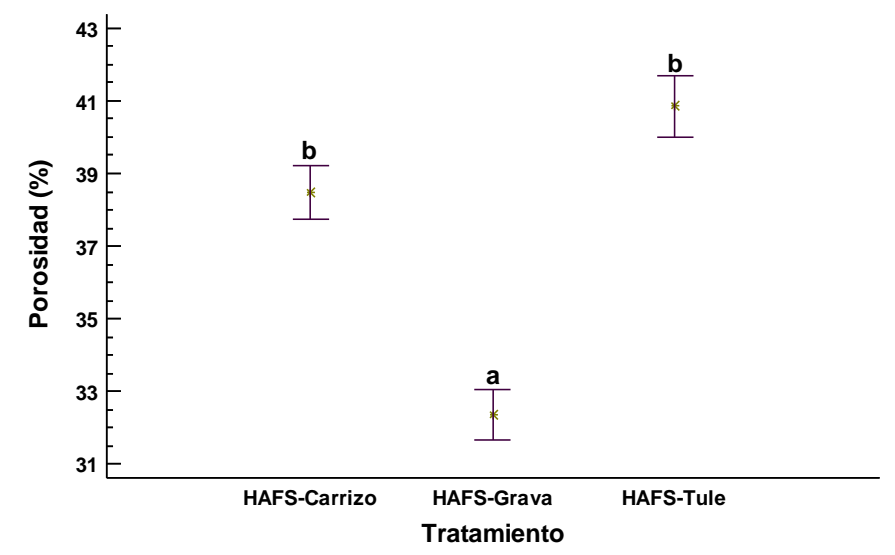

Nota: letras diferentes indican diferencias estadísticamente significativas $(N=12)$.

Fuente: Elaboración propia

\section{Características de las especies}

Los resultados del análisis de la $t$ de Student, comparando las medias de la biomasa de Pontederia sagittata (Tule) y Phragmites australis (Carrizo) usadas en los tratamientos de HAFS-Tule y HAFS-Carrizo después de la poda, indican que existen diferencias estadísticas altamente significativas $(p<0.05)$ (figura 12). El HAFS-Tule obtuvo un promedio en biomasa de vegetación de $40.85 \pm 2.58 \mathrm{~kg}$, mientras que para el HAFS-Carrizo fue $8 \pm 0.73 \mathrm{~kg}$. 
Figura 12. Valores promedios $( \pm \mathrm{EE})$ de la biomasa de las especies $(\mathrm{kg})$

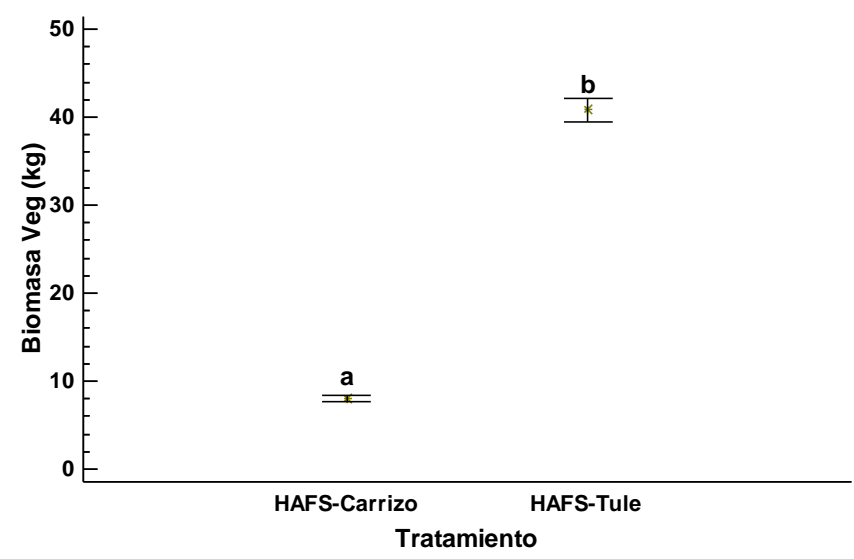

Nota: letras diferentes indican diferencias estadísticamente significativas $(N=12)$.

Fuente: Elaboración propia

Las características de hojas (largo y ancho), altura (m), diámetro y largo de sus raíces, de las cuales se midieron tres a los $0.35 \mathrm{~m}$ de la entrada, tres a los $1.25 \mathrm{~m}$ de en medio y tres más a los $2.15 \mathrm{~m}$ de la salida, presentaron un promedio final que se resume en la tabla 1.

Tabla 1. Mediciones de las especies después de la poda $(N=9)$

\begin{tabular}{|c|c|c|c|c|c|c|c|}
\hline Especie & Distancia $(\mathrm{m})$ & Altura $(\mathrm{m})$ & L. hoja $(\mathrm{cm})$ & A. hoja $(\mathrm{cm})$ & $\varnothing$ tallo $(\mathrm{cm})$ & L. raíz $(\mathrm{cm})$ & Peso $(\mathrm{kg})$ \\
\hline \multirow{2}{*}{$\begin{array}{c}\text { Phragmites } \\
\text { australis }\end{array}$} & 0.35 & $1.65 \pm 0.1$ & $18.92 \pm 2.4$ & $1.57 \pm 0.1$ & $1.78 \pm 0.2$ & $8.80 \pm 1.6$ & $0.18 \pm 0.0$ \\
\cline { 2 - 8 } & 1.25 & $1.64 \pm 0.1$ & $17.97 \pm 7.9$ & $1.65 \pm 0.1$ & $1.69 \pm 0.3$ & $9.43 \pm 0.9$ & $0.18 \pm 0.8$ \\
\hline \multirow{2}{*}{$\begin{array}{c}\text { Pontederia } \\
\text { sagittata }\end{array}$} & 2.15 & $1.54 \pm 0.2$ & $17.62 \pm 8.5$ & $1.63 \pm 0.2$ & $1.66 \pm 0.2$ & $8.60 \pm 1.6$ & $0.18 \pm 0.0$ \\
\cline { 2 - 8 } & 1.25 & $1.29 \pm 0.1$ & $36.21 \pm 0.6$ & $22.50 \pm 2.0$ & $6.53 \pm 0.8$ & $16.05 \pm 1.4$ & $2.41 \pm 0.3$ \\
\cline { 2 - 8 } & 2.15 & $1.32 \pm 0.1$ & $35.96 \pm 1.7$ & $23.71 \pm 1.0$ & $6.58 \pm 0.7$ & $20.00 \pm 3.0$ & $2.50 \pm 0.1$ \\
\hline
\end{tabular}

Nota: Distancia $=$ Distancia longitudinal del HAFS, Peso $=$ Peso de la especie.

Fuente: Elaboración propia

\section{Tiempo de retención hidráulico y remoción de DBO}

Una vez estimado el TRH variando los gastos de operación, se estableció que a 200 L/d los experimentos tendrían 3.1 días de TRH, y se corroboró que a mayor gasto de operación menor TRH, lo que implica una deficiencia de remoción de contaminantes en el HAFS, ya que el contacto del agua con las bacterias y el sistema radicular será menor en los tratamientos (figura 13). 
Figura 13. Tiempos de retención hidráulica contra diferentes caudales

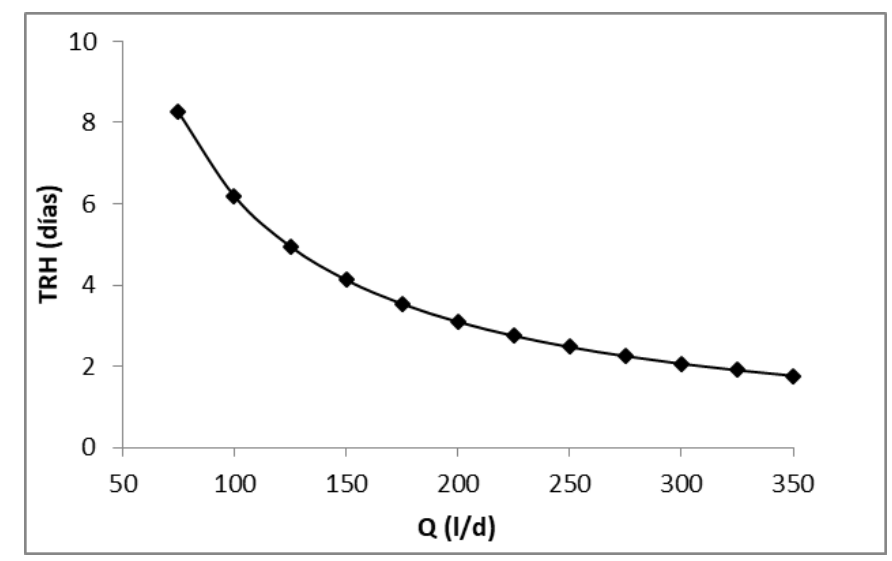

Fuente: Elaboración propia

En la tabla 2 se presenta la remoción y el coeficiente cinético de degradación de la $\mathrm{DBO}_{5}$ en cada uno de los tratamientos. Se observa que las velocidades de reacción son diferentes para cada HAFS con su especie y la degradación de la materia orgánica está relacionada con la concentración de microorganismos en el medio de soporte. Todos los tratamientos fueron con 3.1 días de TRH. El HAFS-Tule antes de la poda en promedio presentó una $k=0.60$ días $^{-1}$ y removió $84.52 \%$ de $\mathrm{DBO}_{5}$; posterior a la poda una $k=1.004$ días $^{-1}$ con $95.4 \%$ de DBO5. El HAFS-Carrizo antes de la poda presentó una $k=0.46$ días $^{-1}$ removiendo $75.73 \%$ de $\mathrm{DBO}_{5}$ y posterior a la poda una $k=0.67$ días $^{-1}$ removiendo $87.47 \%$ de $\mathrm{DBO}_{5}$. El HAFS-Grava presentó antes de la poda una $k=0.21$ días $^{-1}$, removiendo $47.13 \%$ de $\mathrm{DBO}_{5}$, posterior a la poda presentó una $k=0.22$ días $^{-1}$, removiendo $50.01 \%$ de $\mathrm{DBO}_{5}$. Así pues, los tratamientos de Carrizo y Grava requieren de mayor TRH para poder lograr una eficiencia similar a la del HAFS-Tule. 
Revista lberoamericana de las Ciencias Biológicas

y Agropecuarias

Tabla 2. Mediciones de las especies después de la poda $(N=6)$

\begin{tabular}{|c|c|c|c|c|c|c|c|c|}
\hline \multirow{2}{*}{ Tratamiento } & \multicolumn{4}{|c|}{ Antes de la cosecha } & \multicolumn{4}{|c|}{ Después de la cosecha } \\
\hline & Inf. & Efl. & $\operatorname{ER}(\%)$ & $\mathrm{k}\left(\right.$ días $\left.^{-1}\right)$ & Inf. & Efl. & $\mathrm{ER}(\%)$ & $\mathrm{k}\left(\right.$ días $\left.^{-1}\right)$ \\
\hline \multirow{6}{*}{$\begin{array}{c}\text { HAFS- } \\
\text { Tule }\end{array}$} & 415.0 & 63.0 & 84.82 & 0.61 & 393.5 & 23.5 & 94.03 & 0.91 \\
\hline & 338.0 & 60.0 & 82.25 & 0.56 & 430.0 & 15.0 & 96.51 & 1.08 \\
\hline & 436.0 & 65.0 & 85.09 & 0.61 & 415.0 & 16.0 & 96.14 & 1.05 \\
\hline & 395.0 & 49.0 & 87.59 & 0.67 & 380.0 & 20.0 & 94.74 & 0.95 \\
\hline & 410.0 & 61.0 & 85.12 & 0.61 & 440.0 & 15.0 & 96.59 & 1.09 \\
\hline & 360.0 & 64.0 & 82.22 & 0.56 & 390.0 & 22.0 & 94.36 & 0.93 \\
\hline \multirow{6}{*}{$\begin{array}{l}\text { HAFS- } \\
\text { Carrizo }\end{array}$} & 415.0 & 98.0 & 76.39 & 0.47 & 393.5 & 59.3 & 84.93 & 0.61 \\
\hline & 338.0 & 90.0 & 73.37 & 0.43 & 430.0 & 53.5 & 87.56 & 0.67 \\
\hline & 436.0 & 95.0 & 78.21 & 0.49 & 415.0 & 43.7 & 89.47 & 0.73 \\
\hline & 395.0 & 89.0 & 77.47 & 0.48 & 380.0 & 48.1 & 87.34 & 0.67 \\
\hline & 410.0 & 91.0 & 77.80 & 0.49 & 440.0 & 44.2 & 89.95 & 0.74 \\
\hline & 360.0 & 104.0 & 71.11 & 0.40 & 390.0 & 56.3 & 85.56 & 0.62 \\
\hline \multirow{6}{*}{$\begin{array}{l}\text { HAFS- } \\
\text { Grava }\end{array}$} & 415.0 & 220.5 & 46.87 & 0.20 & 393.5 & 212.0 & 46.12 & 0.20 \\
\hline & 338.0 & 210.5 & 37.72 & 0.15 & 430.0 & 206.5 & 51.98 & 0.24 \\
\hline & 436.0 & 195.7 & 55.11 & 0.26 & 415.0 & 196.5 & 52.65 & 0.24 \\
\hline & 395.0 & 220.3 & 44.23 & 0.19 & 380.0 & 210.0 & 44.74 & 0.19 \\
\hline & 410.0 & 202.2 & 50.68 & 0.23 & 440.0 & 205.0 & 53.41 & 0.25 \\
\hline & 360.0 & 186.5 & 48.19 & 0.21 & 390.0 & 190.5 & 51.15 & 0.23 \\
\hline
\end{tabular}

Nota: Inf. $=$ Influente, Efl. $=$ Efluente, ER $(\%)=$ Eficiencia de remoción.

Fuente: Elaboración propia

\section{Discusión}

Los microorganismos antes y después de la poda presentan mayor concentración en el testigo con respecto a los humedales con especies y en el HAFS-Tule mostraron una diferencia de $9487 \mathrm{mg} / \mathrm{kg}$, en el HAFS-Carrizo presentaron una diferencia de $1696.9 \mathrm{mg} / \mathrm{kg}$ y en el caso del HAFS-Grava la diferencia fue de $-1105.1 \mathrm{mg} / \mathrm{kg}$. En este último hay un incremento de la biomasa microbiana al medio de soporte (fijación y adaptación), pues no cuenta con raíces o plantas. Es más notorio el cambio de microorganismos en el HAFS-Tule 
Revista Iberoamericana de las Ciencias Biológicas y Agropecuarias

por ser una planta macrófita de mayor densidad, pues el tallo y la raíz dan lugar a sitios para una mayor fijación de microorganismos y cuando es podada se muestra una disminución de la población microbiana. Este efecto no es igual en el HAFS-Carrizo debido a que los microorganismos no dependen exclusivamente de las plantas, de manera tal que es menor el efecto en los microorganismos después de la poda. Autores como Li et al. (2013) establecen que en $P$. australis, aunque puede presentar mayor diversidad bacteriana en la raíz con respecto a Typha angustifolia L., estas se involucran en el ciclo total del $N$ a diferencia de las bacterias de la Typha, que se involucran en la eliminación de $P$ y materia orgánica. Los microorganismos en el HAFS-Tule disminuyen después de la poda y puede atribuirse a que en la zona superior del humedal hay oxígeno liberado por las raíces de las plantas y causa su disminución por la poda de las especies (Delgadillo, Camacho, Pérez y Andrade, 2010).

Las plantas, al transferir oxígeno desde la atmósfera a través de hojas y tallos, lo liberan en las raíces creando regiones aerobias, donde los microorganismos utilizan el oxígeno disponible para la degradación de materia orgánica y la nitrificación, por lo que al podar las especies se modifican las microzonas aeróbicas en la superficie de las raíces y los rizomas (EPA, 2000; Rahman et al., 2020). Las poblaciones microbianas, por la variación del oxígeno, afectan la degradación de la materia orgánica, aunque autores como Herzog et al. (2021) mencionan que los efectos que la poda tiene en la mejora de la calidad del agua siguen siendo desconocidos para ciertos contaminantes. En los HAFS parte de la carga orgánica se concentra en la entrada, por lo que, de acuerdo con Delgadillo et al. (2010), los microorganismos heterótrofos requieren material orgánico como fuente de energía y carbono para la síntesis de nuevos microorganismos, lo que hace notar una mayor biomasa de microorganismos a la entrada del humedal que va disminuyendo en función a la longitud del humedal como consecuencia de la disminución de la materia orgánica hacia el efluente.

La humedad mostró disminuciones; el mayor cambio en la diferencia del promedio se presentó en el HAFS-Grava (3.36\%), seguido del HAFS-Carrizo (2.75\%) en sus medianas, y la diferencia menor en el promedio en el HAFS-Tule (1.49\%) después de la poda. Estas diferencias son atribuidas a las especies, ya que las plantas protegen y evitan el calentamiento del medio de soporte manteniendo la humedad promedio. Tanner y Headley (2011) evaluaron la temperatura del agua en el tratamiento de HA y concluyeron que, debido a la sombra que ejercen las plantas, la temperatura en el agua puede llegar a ser menor que la temperatura ambiente en promedio de $2{ }^{\circ} \mathrm{C} \mathrm{a} 4{ }^{\circ} \mathrm{C}$, esto es, más fresca. Por lo que después 
Revista Iberoamericana de las Ciencias Biológicas y Agropecuarias

de podar, la radiación solar calienta la grava de manera directa, aumenta la temperatura y por consiguiente evapora el agua del estrato superior, todo lo cual lleva a la disminución de la humedad en la parte superior de los HAFS.

La porosidad del HAFS-Carrizo incrementó $3.4 \%$ después de la poda, en el HAFSTule el incremento fue de $2 \%$ y en el HAFS-Grava disminuyó $1.1 \%$. Esta porosidad, aunque es mínima y temporal, se debe al espacio que van dejando las raíces de las especies después de la poda, pues inician nuevamente su crecimiento y estabilización; por otro lado, hay un efecto por la cosecha de aquellas especies que han cumplido con su ciclo de vida y tienen que ser retiradas en el mantenimiento preventivo (Conagua, 2016). Para el caso del HAFSGrava, la disminución se debe al acomodo propio de las partículas en el lecho. Es importante mencionar que la porosidad de los medios de soporte se verá reducida conforme pasa el tiempo de operación debido a la sedimentación y filtración de sólidos en suspensión, la creación de biopelículas en las partículas del material de filtración, la precipitación química y el crecimiento de raíces y rizomas de la vegetación (Vymazal, 2018), motivo por el cual es necesario aplicar un eficiente tratamiento primario, usar de materiales porosos adecuados, mantener cargas adecuadas de sustancias orgánicas y sólidos en suspensión para ralentizar enormemente el proceso de obstrucción (Conagua, 2016; Wang, Sheng y Xu, 2021).

Con los parámetros obtenidos del medio de soporte se estimó que el HAFS-Carrizo permite un volumen de agua de $0.55 \pm 0.04 \mathrm{~m}^{3}$ y $0.95 \pm 0.01 \mathrm{~m}^{3}$ de grava, una conductividad hidráulica (Ks) de $321.31 \mathrm{~m}^{3} / \mathrm{m}^{2}$ d y una velocidad de flujo (v) de $0.32 \mathrm{~m} / \mathrm{d}$, mientras que el HAFS-Grava contiene $0.49 \pm 0.01 \mathrm{~m}^{3}$ de agua con $1.01 \pm 0.00 \mathrm{~m}^{3}$ de grava, $\mathrm{Ks}=375.44$ $\mathrm{m}^{3} / \mathrm{m}^{2} \mathrm{~d}$ y $\mathrm{v}=0.38 \mathrm{~m} / \mathrm{d}$ y el HAFS-Tule almacena $0.60 \pm 0.02 \mathrm{~m}^{3}$ de agua y $0.90 \pm 0.01 \mathrm{~m}^{3}$ de grava, $\mathrm{Ks}=303.24 \mathrm{~m}^{3} / \mathrm{m}^{2} \mathrm{~d} \mathrm{y} \mathrm{v}=0.30 \mathrm{~m} / \mathrm{d}$. En los tres tratamientos la conductividad hidráulica y la velocidad del flujo (> $36.0 \mathrm{~m} / \mathrm{d}$ para grava) cumplen con los niveles recomendados por Rahman et al. (2020) y la Conagua (2016). Es importante dar seguimiento a estas variables durante un tiempo prolongado (de uno a dos años), pues, de acuerdo con Hoffmann et al. (2011), a largo plazo estos parámetros cambian y disminuyen debido al desarrollo de las raíces de las plantas y a la acumulación de residuos no degradables que se quedan en el medio de soporte. En los filtros sin plantas el suelo tiene que ser tratado para recuperar su conductividad hidráulica (sacando la capa superficial del sustrato). El sistema de raíces mantiene la conductividad hidráulica del sustrato de arena gruesa, por lo que las plantas adecuadas con un sistema bien desarrollado de raíces y rizoma desempeñan un papel 
Revista Iberoamericana de las Ciencias Biológicas y Agropecuarias

importante en el mantenimiento y restauración de la conductividad del lecho filtrante (Conagua, 2016).

Los micrófitos empleados en HA presentan variación en la remoción de materia orgánica y coeficientes cinéticos. La cinética de reacción de primer orden es en gran medida la que representa la eliminación de contaminantes orgánicos $(\mathrm{C}, \mathrm{N}, \mathrm{P})$ para HAFS (Cui et al., 2011; Wang, Bo y Liu, 2015). Quintero (2014), reportó que la $\mathrm{DBO}_{5}$ se removió favorablemente en HAFS con Heliconia Psittacorum, con más de $87 \%$ a TRH de 1.6 días. En nuestro estudio, todos los tratamientos fueron con 3.1 días de TRH. El $\mathrm{DBO}_{5}$ en el influente fue de $408.1 \mathrm{mg} / \mathrm{L}$ con una temperatura del agua $27.63{ }^{\circ} \mathrm{C}$. Y el mejor tratamiento fue el HAFS-Tule, que removió $95.44 \%$ de $\mathrm{DBO}_{5}(18.6 \mathrm{mg} / \mathrm{L}$ en el efluente) y un coeficiente cinético de degradación (k) de 1.004 días $^{-1}$, cumpliendo el límite máximo permisible en la NOM-001-Semarnat-1996 de 30 mg/L en el promedio diario para protección de vida acuática en ríos. El HAFS-Carrizo removió $87.5 \%$ (50.85 mg/L en el efluente) con una $\mathrm{k}=0.66$ días $^{-}$ 1. De manera similar, en el HAFS-Grava se removió $50.1 \%$ (203.44 mg/L en el efluente), con una $\mathrm{k}=0.22$ días $^{-1}$. Las constantes cinéticas (k) que reportan la EPA (2000) y Crites y Tchobanoglous (2000) para HAFS van de 0.86 a 1.84 días $^{-1}\left(20^{\circ} \mathrm{C}\right)$ y estas también dependen del medio se soporte utilizado recomendando para grava $\mathrm{k}=0.86$ días $^{-1}\left(20{ }^{\circ} \mathrm{C}\right)$, y si se utilizan estas constantes para el diseño debe ajustarse la $\mathrm{k}$ a temperaturas ambientes (Arrhenius) y evaluarse a condiciones normales de operación (Conagua, 2016). Otro estudio en el sureste de México es el de López et al. (2019), quienes evaluaron un HAFS tratando agua residual doméstica (204 \pm 66 L/día, 377 mg/L de $\left.\mathrm{DBO}_{5}\right)$ con Thalia geniculata y obtuvieron $85.6 \%$ de remoción de $\mathrm{DBO}_{5}$ con una $\mathrm{k}=0.43$ días $^{-1}$, y aunque las constantes son diferentes (menor a la de nuestro estudio), el desempeño en la remoción de la DBO es similar. Dicha eficiencia se atribuye a la temperatura de operación $\left(27^{\circ} \mathrm{C}\right)$ y a mayor TRH (4.2 días).

Los microorganismos adheridos a la superficie se consideran responsables de las reacciones biológicas en el sustrato sumergido y las tasas de reacción microbiana de los HAFS pueden ser mayores que las de HAFL para muchos contaminantes. Por ello, un HAFS puede tener una menor superficie que un HAFL para los mismos caudales y calidad del agua (Conagua, 2016; Rahman et al., 2020). Los HAFS, al carecer de oxígeno, limitan la remoción del amoniaco (nitrificación), pero aun así el sistema es efectivo en la remoción de DBO (95.44 \% para Tule y $87.5 \%$ con Carrizo), algunos contaminantes como metales y orgánicos 
prioritarios, dado que el tratamiento es bajo condiciones aeróbicas y anóxicas (Herzog et al., 2021).

Finalmente, aunque es notoria la diferencia de estructura y biomasa recuperada de la cosecha de $P$. australis y $P$. sagittata, no se muestran diferencias entre las plantas a lo largo de cada HAFS $(0.35 \mathrm{~m}, 1.25 \mathrm{~m}$ y $2.15 \mathrm{~m})$; en promedio, una planta de $P$. australis presenta una altura $1.61 \mathrm{~m}$, longitud de hoja de $18.17 \mathrm{~cm}$, ancho de hoja de $1.62 \mathrm{~cm}$, diámetro de 1.71 $\mathrm{cm}$, largo de raíz de $8.94 \mathrm{~cm}$ y un peso $0.18 \mathrm{~kg}$. Mientras que $P$. sagittata presentó en promedio una altura $1.33 \mathrm{~m}$, longitud de hoja de $35.23 \mathrm{~cm}$, ancho de hoja de $22.34 \mathrm{~cm}$, diámetro de $6.20 \mathrm{~cm}$, largo de raíz de $18.79 \mathrm{~cm}$ y un peso $2.71 \mathrm{~kg}$. Se ha reportado que las remoción de contaminantes se reduce cuando no se realiza una poda sistemática de la vegetación y se retira el material podado a sitios fuera de los HA, por lo que se debe realizar con una frecuencia de dos a tres veces por año y eliminar principalmente la vegetación con apariencia amarillenta (vegetación de más edad), muertas y secas; en cada poda, eliminar entre $10 \%$ y $15 \%$ del total de la vegetación en las celdas evitando que se reincorporen los nutrientes al humedal (Vymazal y Kröpfelová, 2008).

\section{Conclusión}

Los resultados presentes son un de humedal joven (un año operación) y se encontró que los microorganismos en el HAFS-Tule después de la poda se redujeron $22.09 \%$. Esta especie brinda mayor densidad radicular para el establecimiento de los microorganismos. En el HAFS-Carrizo es mucho menor el impacto, solo se redujo $3.8 \%$, y en el HAFS-Grava se incrementaron en $1.89 \%$ los microorganismos, posiblemente por su adaptación al sustrato y a la mayor tolerancia del sol.

En los tres HAFS se presentó una disminución gradual de la entrada a la salida de los microorganismos, comportándose conforme disminuye la carga orgánica a lo largo del humedal.

El tratamiento más eficiente después de la poda en la remoción de $\mathrm{DBO}_{5}$ fue el HAFSTule, que removió $95.44 \%$ de $\mathrm{DBO}_{5}$ con una $\mathrm{k}=1.004$ días $^{-1}$, seguido del HAFS-Carrizo, con $87.5 \%$ y una $\mathrm{k}=0.67$ días $^{-1}$. Y el HAFS-Grava removió $50.1 \%$ con una $\mathrm{k}=0.22$ días $^{-1}$. Esto muestra que sí se incrementó 10.88 \% respecto al antes y después de la eliminación de materia orgánica con Potendenria sagittata y $11.74 \%$ con P. australis. 


\section{Futuras líneas de investigación}

Para conocer el potencial del efecto de la poda en HA se debe continuar el estudio en los reactores con otras especies macrófitas en un corto plazo y evaluar los tiempos de retención hidráulica para poder remover nutrientes y otros xenobióticos en valores de concentración que permitan descargar a cuerpos receptores de forma segura. Otra línea de investigación a mediano plazo sería evaluar la eficiencia de remoción con aguas de concentración media y fuerte con diferentes especies macrófitas, de manera tal que se pueda recomendar los HA para diferentes casos eficientes y económicos.

\section{Referencias}

Araujo, L., Molina, S. y Noguera, L. (2018). Aprovechamiento de los lodos provenientes de plantas de tratamiento de aguas residuales como materia prima en la industria de la construcción: revisión bibliográfica. Revista Agunkuyaa, 8(1), 21-28 . Recuperado de https://doi.org/10.33132/27114260.1231.

Asprilla, W. J., Ramírez, J. S. y Rodriguez, D. C. (2020). Humedales artificiales de flujo subsuperficial: comparación de metodologías de diseño para el cálculo del área superficial basado en la remoción de materia orgánica. Ingenierías USBMed, 11(2), 65-73,

Castañeda, A. A. y Flores, H. E. (2013). Tratamiento de aguas residuales domésticas mediante plantas macrófitas típicas en Los Altos de Jalisco, México. Paakat: Revista de Tecnología y Sociedad, 3(5). Recuperado de http://www.udgvirtual.udg.mx/paakat/index.php/paakat/article/view/208/298.

Comisión Nacional del Agua [Conagua]. (2016). Manual de agua potable, alcantarillado y saneamiento. Diseño de plantas de tratamiento de aguas residuales municipales: humedales artificiales. Ciudad de México, México: Secretaría de Medio Ambiente y Recursos Naturales. Recuperado de http://cmx.org.mx/wpcontent/uploads/MAPAS\%202015/libros/SGAPDS-1-15-Libro30.pdf.

Comisión Nacional del Agua [Conagua]-Secretaría de Medio Ambiente y Recursos Naturales [Semarnat]. (2019). Inventario nacional de plantas municipales de potabilización y de tratamiento de aguas residuales en operación. Recuperado de https://www.gob.mx/cms/uploads/attachment/file/611037/Inventario_2019.pdf. 
Cui, L. J., Zhang, Y., Zhao, X. S., Li, W., Zhang, M. Y., Wang, Y. F., and Li, S. N. (2011). Pollutants removal in subsurface constructed wetland based on the first-order kinetic model. China Environmental Science, 31(10), 1697-1704.

Crites, R. y Tchobanoglous, G. (2000). Sistemas de manejo de aguas residuales para núcleos pequeños y descentralizados. Colombia: McGraw-Hill.

Delgadillo, O., Camacho, A., Pérez, L. F. y Andrade, M. (2010). Depuración de aguas residuales por medio de humedales artificiales. Cochabamba, Bolivia: Centro Andino para la Gestión y Uso del Agua.

Environmental Protection Agency [EPA]. (2000). Folleto informativo de tecnología de aguas residuales. Humedales de flujo subsuperficial. EPA 832- F-00-023. Washington, United States: Environmental Protection Agency.

García, J. A., López, G. y Torres, C. A. (2019) Evaluación de medios de soporte en humedales artificiales con vegetación Sagittaria latifolia en la remoción de contaminantes básicos. CIBA. Revista Iberoamericana de las Ciencias Biológicas y Agropecuarias, 8(15), 16-51.

Gallegos, J., López, G., Bautista, R. G. and Torres, C. A. (2018). Evaluation of free flow constructed wetlands with Sagittaria latifolia and Sagittaria lancifolia in domestic wastewater treatment. Ingeniería Agrícola y Biosistemas, 10(2), 49-65.

Herzog, T., Mehring, A., Hatt, B., Ambrose, R., Levin, L. and Winfrey, B. (2021). Pruning stormwater biofilter vegetation influences water quality improvement differently in Carex appressa and Ficinia nodosa. Urban Forestry \& Urban Greening, 59. Retrieved from https://doi.org/10.1016/j.ufug.2021.127004.

Hoffmann, H., Platzer, C., Winker, M. y von Muench, E. (2011). Revisión técnica de humedales artificiales de flujo subsuperficial para el tratamiento de aguas grises y aguas domésticas. Eschborn, Alemania: Agencia de Cooperación Internacional de Alemania- Programa de Saneamiento Sostenible. https://www.susana.org/_resources/documents/default/2-1235-giz2011-technologyreview-constructed-wetlands-in-spanish.pdf.

Li, Y. H, Zhu, J. N., Liu, Q. F., Liu, Y., Liu, M., Liu, L. and Zhang Q. (2013). Comparison of the diversity of root-associated bacteria in Phragmites australis and Typha 
Revista Iberoamericana de las Ciencias Biológicas

y Agropecuarias

angustifolia L. in artificial wetlands. World Journal of Microbiology and Biotechnology, 29(8), 149.9-508.

López, G., Torres, C. A., Bautista, R. G., Hernández, J. R., Cruz, E. y Ferrer, M. I. (2014). Diseño de sistemas experimentales de humedales artificiales de flujo libre y subsuperficial. En Perspectiva Científica desde la UJAT (pp. 133-146). Villahermosa, México: Universidad Juárez Autónoma de Tabasco. Recuperado de http://www.archivos.ujat.mx/2014/divulgacion\%20cientifica/SDDC2013final.pdf.

López, G., Bautista, R. G., Valdés, A., Torres, C. A., López, R., Pérez, E. and Pampilón, L. (2019). Spatial distribution behavior of basic pollutants in a subsurface-flow wetland with Thalia geniculata. International Journal of Environmental Impacts, 2(2), 145160.

Mancipe, L. M. y Triviño, M. (2018). Valoración de lodos de planta de tratamiento de aguas residuales (PTAR) como materia prima para la extracción de lípidos en la obtención de biodiésel. Revista Ion, 31(1), 71-79. Recuperado de http://dx.doi.org/10.18273/revion.v31n1-2018012.

Miranda, E., Sandoval, L., Calvo, Y., Moeller, G. E. y Sarracino, O. (2017). Evaluación del proceso de coagulación-floculación para el tratamiento de un efluente de la industria textil. Jeeos, 1(3), 43-54.

Muñoz, D. J., Soler, A., López, F. y Hernádez, M. M. (2015). Edafología: manual de métodos de análisis del suelo. Ciudad de México, México: Editorial FESI, UNAM.

Quintero, J. A. (2014). Evaluación de humedales artificiales pilotos de flujo horizontal y tipo superficial y subsuperficial para el tratamiento de aguas residuales. Ingenium, 15(29), 85-112. Recuperado de https://doi.org/10.21500/01247492.1347.

Rangel, J. G., Mendivil, K., Cedillo, C. I. G., Rochín, J. J., Rodríguez, A. E. and Bustos, Y. A. (2019). Optimization of organic matter degradation kinetics and nutrient removal on artificial wetlands using Eichhornia crassipes and Typha domingensis. Environmental Technology, 40(5), 633-641.

Rahman, M. E., Bin Halmi, M. I. E., Bin Abd Samad, M. Y., Uddin, M. K., Mahmud, K., Abd Shukor, M. Y., Sheikh Abdullah, S. R. and Shamsuzzaman, S. M. (2020). Design, Operation and Optimization of Constructed Wetland for Removal of Pollutant. International Journal of Environmental Research and Public Health, 17(22), 8339. Retrieved from https://doi.org/10.3390/ijerph17228339. 
Secretaría de Economía. (2001). NMX-AA-028-SCFI-2001. Análisis de agua Determinación de la demanda bioquímica de oxígeno en aguas naturales, residuales $\left(\mathrm{DBO}_{5}\right)$ y residuales tratadas - Método de prueba.

Secretaría de Medio Ambiente, Recursos Naturales [Semarnat]. (24 de junio de 1996). NOM001-Semarnat-1996. Establece los límites máximos permisibles de contaminantes en las descargas de aguas residuales en aguas y bienes nacionales. Diario Oficial de la Federación.

Secretaría de Medio Ambiente, Recursos Naturales [Semarnat]. (7 de diciembre de 2001). NOM-021-Recnat-2000. Que establece las especificaciones de fertilidad, salinidad y clasificación de suelos. Estudio, muestreo y análisis. Diario Oficial de la Federación.

Tanner, C. C. and Headley, T. (2011). Components of floating emergent macrophyte treatment wetlands influencing removal of stormwater pollutants. Ecological Engineering, 37, 474-486.

Torres, C. A., López, G., Romellón, M. J., Vázquez, M. B. y Comparán, L. E. (2020). Biomasa de origen vacuno en la remoción de contaminantes básicos en un reactor discontinuo secuencial. CIBA Revista Iberoamericana de las Ciencias Biológicas y Agropecuarias, 9(18), 1-32. Recuperado de https://doi.org/10.23913/ciba.v9i18.98.

Vázquez, M. B., López, G. (2011). Evaluación Técnica de un Tanque Imhoff para el tratamiento de aguas residuales en Centro, Tabasco. U. Tecnociencia. 5 (1) 32 - 47.

Vymazal, J. and Kröpfelová, L. (2008). Environment pollution 14. Wastewater treatment inconstructed wetlands with horizontal sub-surface flow. (Ed.) Springer pp. 566 .

Vymazal, J. (2018). Does clogging affect long-term removal of organics and suspended solids in gravel-based horizontal subsurface flow constructed wetlands? Chemical Engineering Journal, 331, 663-674. Retrieved from https://doi.org/10.1016/j.cej.2017.09.048.

Wang, H., Bo, G. and Liu, X. (2015). Chemical reaction kinetics analysis of removing phosphorus pollutants from wastewater in different constructed wetlands. Fresenius Environmental Bulletin, 24(2), 596-601.

Wang, H., Sheng, L. and Xu, J. (2021). Clogging mechanisms of constructed wetlands: A critical review. Journal of Cleaner Production, 295. Retrieved from https://doi.org/10.1016/j.jclepro.2021.126455. 


\section{Mario José Romellón Cerino}

Candidato a doctor en Ciencias Ambientales por la Universidad de Xalapa, maestro en Ingeniería y Protección Ambiental e ingeniero Ambiental por la Universidad Juárez Autónoma de Tabasco (UJAT). Es profesor-investigador de tiempo completo en el Instituto Tecnológico de Villahermosa (ITVH) del Tecnológico Nacional de México (TecNM). Miembro del cuerpo académico "Innovación en el mejoramiento ambiental”, miembro del consejo de posgrado de la maestría en Ingeniería del ITVH y responsable del Laboratorio de Química Pesada del ITVH.

\section{Gaspar López Ocaña}

Doctor en Ciencias en Ecología y Manejo de Sistemas Tropicales, maestro en Ingeniería y Protección Ambiental e ingeniero Ambiental, todos los grados por la Universidad Juárez Autónoma de Tabasco (UJAT). Es profesor-investigador de tiempo completo, miembro del cuerpo académico "Ingeniería y tecnología ambiental" y responsable del Laboratorio de Tecnología del Agua de la División Académica de Ciencias Biológicas (DACBiol) de la UJAT.

\section{José Reyes Osorio}

Maestro en Ingeniería y Protección Ambiental por la Universidad Juárez Autónoma de Tabasco (UJAT), ingeniero Químico por el Instituto Tecnológico de Villahermosa (ITVH) del Tecnológico Nacional de México (TecNM). Es profesor de tiempo completo en el ITVH y miembro del cuerpo académico "Innovación en el mejoramiento ambiental".

\section{Julio César Romellón Cerino}

Doctor en Desarrollo Tecnológico por la Universidad de Ciencia y Tecnología Descartes, maestro en Administración por la Universidad Juárez Autónoma de Tabasco (UJAT) e ingeniero Industrial Químico por el Instituto Tecnológico de Villahermosa (ITVH) del Tecnológico Nacional de México (TecNM). Profesor investigador de tiempo completo en el ITVH. 
Revista Iberoamericana de

las Ciencias Biológicas

y Agropecuarias

\section{Víctor Manuel Arias Peregrino}

Doctor en Desarrollo Tecnológico por la Universidad de Ciencia y Tecnología Descartes, maestro en Tecnologías de la Computación por la Universidad Mundo Maya e ingeniero en Sistemas Computacionales por el Instituto Tecnológico de Villahermosa (ITVH) del Tecnológico Nacional de México (TecNM). Es docente de tiempo completo y secretario del consejo de posgrado en Tecnologías de la Información en el ITVH.

\begin{tabular}{|c|c|}
\hline Rol de contribución & Autor(es) \\
\hline Conceptualización & Mario José Romellón Cerino 50 \%, Gaspar López Ocaña 50 \% \\
\hline Metodología & Mario José Romellón Cerino 50 \%, Gaspar López Ocaña 50 \% \\
\hline Software & $\begin{array}{l}\text { Mario José Romellón Cerino } 20 \text { \%, Victor Manuel Arias Peregrino } \\
20 \% \text {, Julio Cesar Romellón Cerino } 20 \% \text {, José Reyes Osorio } 20 \% \text {, } \\
\text { Gaspar López Ocaña } 20 \%\end{array}$ \\
\hline Validación & $\begin{array}{l}\text { Mario José Romellón Cerino } 20 \text { \%, Victor Manuel Arias Peregrino } \\
20 \% \text {, Julio Cesar Romellón Cerino } 20 \% \text {, José Reyes Osorio } 20 \% \text {, } \\
\text { Gaspar López Ocaña } 20 \%\end{array}$ \\
\hline Análisis formal & $\begin{array}{l}\text { Mario José Romellón Cerino } 20 \text { \%, Victor Manuel Arias Peregrino } \\
20 \% \text {, Julio Cesar Romellón Cerino } 20 \% \text {, José Reyes Osorio } 20 \% \text {, } \\
\text { Gaspar López Ocaña } 20 \%\end{array}$ \\
\hline Investigación & Mario José Romellón Cerino 50 \%, Gaspar López Ocaña 50 \% \\
\hline Recursos & Gaspar López Ocaña 100 \% \\
\hline Curación de datos & $\begin{array}{l}\text { Mario José Romellón Cerino } 20 \text { \%, Victor Manuel Arias Peregrino } \\
20 \% \text {, Julio Cesar Romellón Cerino } 20 \% \text {, José Reyes Osorio } 20 \% \text {, } \\
\text { Gaspar López Ocaña } 20 \%\end{array}$ \\
\hline
\end{tabular}


Revista Iberoamericana de las Ciencias Biológicas y Agropecuarias

\begin{tabular}{|l|l|}
\hline $\begin{array}{l}\text { Escritura. Preparación } \\
\text { del borrador original }\end{array}$ & $\begin{array}{l}\text { Mario José Romellón Cerino 20 \%, Victor Manuel Arias Peregrino } \\
20 \%, \text { Julio Cesar Romellón Cerino 20 \%, José Reyes Osorio 20 \%, } \\
\text { Gaspar López Ocaña } 20 \%\end{array}$ \\
\hline $\begin{array}{l}\text { Escritura. Revisión y } \\
\text { edición }\end{array}$ & $\begin{array}{l}\text { Mario José Romellón Cerino 20\%, Victor Manuel Arias Peregrino } \\
20 \%, \text { Julio Cesar Romellón Cerino 20\%, José Reyes Osorio 20\%, } \\
\text { Gaspar López Ocaña } 20 \%\end{array}$ \\
\hline $\begin{array}{l}\text { Visualización } \\
\text { Mario José Romellón Cerino 20 \%, Victor Manuel Arias Peregrino } \\
20 \%, \text { Julio Cesar Romellón Cerino 20 \%, José Reyes Osorio 20 \%, } \\
\text { Gaspar López Ocaña 20 \% }\end{array}$ \\
\hline $\begin{array}{l}\text { Supervisión } \\
\text { proyectos }\end{array}$ & $\begin{array}{l}\text { Mario José Romellón Cerino } 50 \%, \text { Gaspar López Ocaña 50 \% } \\
\text { Adquisición de fondos }\end{array}$ \\
\hline
\end{tabular}

